\title{
Acute and Chronic Ethanol Alter Glutamatergic Transmission in Rat Central Amygdala: an In Vitro and In Vivo Analysis
}

\author{
Marisa Roberto, Paul Schweitzer, Samuel G. Madamba, David G. Stouffer, Loren H. Parsons, and George R. Siggins \\ Department of Neuropharmacology, The Scripps Research Institute, La Jolla, California 92037
}

\begin{abstract}
The modulation of glutamatergic transmission by ethanol may contribute to ethanol intoxication, reinforcement, tolerance, and dependence. Therefore, we used in vitro electrophysiological and in vivo microdialysis techniques to investigate the effects of acute and chronic ethanol on glutamatergic transmission in the central nucleus of amygdala (CeA). Superfusion of 5- 66 mm ethanol decreased compound glutamatergic EPSPs and EPSCs in CeA neurons, with half-maximal inhibition elicited by $14 \mathrm{~mm}$ ethanol. Ethanol (44 mM) decreased both non-NMDAR- and NMDAR-mediated EPSPs and EPSCs by $21 \%$. Both the ethanol- and ifenprodil-induced depression of NMDARmediated EPSPs and EPSCs was enhanced in rats that received chronic ethanol treatment (CET). Ifenprodil also occluded the ethanol effect, suggesting that NR2B subunit-containing receptors may be involved. With local applications of NMDA, acute ethanol elicited a greater inhibition of NMDA currents in slices taken from CET (47\%) compared with naive (30\%) animals, suggesting that CET sensitizes NMDA receptors to ethanol. Acute ethanol also reduced paired pulse facilitation of EPSPs and EPSCs only in CET animals, suggesting acute ethanol-induced increase of glutamate release. This finding was supported by in vivo experiments showing that infusion of ethanol $(0.1-1 \mathrm{M})$ via reverse microdialysis significantly increased glutamate release into the CeA dialysate but only after CET. Moreover, baseline CeA glutamate content was significantly higher in CET compared with naive animals. These combined findings suggest that CET and withdrawal lead to neuroadaptations of glutamatergic transmission at both presynaptic and postsynaptic sites in CeA, and glutamatergic synapses in CeA may play an important role in ethanol dependence.
\end{abstract}

Key words: alcohol; electrophysiology; microdialysis; chronic alcohol treatment; ethanol dependence; paired pulse facilitation; NMDA

\section{Introduction}

Alcoholism is a complex behavioral disorder characterized by excessive consumption of ethanol, the development of tolerance and dependence, and the impairment of social and occupational functioning (Tabakoff and Hoffman, 1996; Koob et al., 1998; Tsai and Coyle, 1998). Alterations in an individual's control over alcohol intake can arise as a consequence of adaptive and compensatory changes in the brain produced by the chronic presence of ethanol. Many studies using a variety of experimental techniques indicate that the amygdaloid complex plays a crucial role in drug dependence and ethanol-reinforcing actions (Davis et al., 1994; Koob et al., 1998; Koob and Le Moal, 2001). The central nucleus of amygdala (CeA) is considered particularly important in mediating the behavioral effects of ethanol (Rassnick et al., 1993; Hyytia and Koob, 1995; Pich et al., 1995; Roberts et al., 1996; Eckardt et al., 1998; Koob et al., 1998).

There is considerable evidence that glutamate-mediated exci-

Received Sept. 4, 2003; revised Dec. 13, 2003; accepted Dec. 19, 2003.

This work was supported by National Institutes of Health Grants AA013517, AA06420, DA03665, DA13658, and AA12294. We thank Dr. G. F. Koob, Dr. J. R. Henriksen, and Dr.P.J. Kenny for critical comments on this manuscript and Dr. W. Fröstl and Dr. A. Suter (Novartis Pharma) for CGP-55854A.

Correspondence should be addressed to Dr. George R. Siggins, CVN-12, The Scripps Research Institute, 10550 North Torrey Pines Road, La Jolla, CA 92037. E-mail: geobob@scripps.edu.

DOI:10.1523/JNEUROSCI.5077-03.2004

Copyright $\odot 2004$ Society for Neuroscience $\quad$ 0270-6474/04/241594-10\$15.00/0 tatory neurotransmission plays an important role in mediating the behavioral actions of acutely administered ethanol and the neuroadaptations associated with prolonged ethanol exposure that underlie ethanol dependence (Eckardt et al., 1998) (for review, see Krystal et al., 2003). The ionotropic glutamate receptors are classified into three subtypes: NMDA, AMPA, and kainate receptors. Although the molecular mechanisms remain to be elucidated, there is a broad consensus that both NMDA and nonNMDA (AMPA and kainate) glutamate receptors are inhibited by ethanol in several brain regions (Hoffman et al., 1989; Lovinger et al., 1989; Nie et al., 1994; Martin et al., 1995; Tabakoff and Hoffman, 1996; Calton et al., 1998; Tsai and Coyle, 1998; Woodward, 1999; Kumari and Ticku, 2000; Criswell et al., 2003). It has been demonstrated that NR2A and NR2B subunit-containing NMDA receptors (NMDARs) are especially sensitive to ethanol inhibition (Masood et al., 1994). There also is evidence that the activation of AMPA and kainate and NMDARs within the CeA plays a crucial role in the negative affective component of morphine abstinence (Vanderschuren and Kalivas, 2000; Watanabe et al., 2002). In addition, amygdala lesions (Post et al., 1987; Wolf et al., 1995) or injection of an NMDA antagonist into the amygdala (Kalivas and Alesdatter, 1993; Robledo et al., 1996) prevented behavioral sensitization to cocaine or amphetamine.

Importantly, the effects of ethanol on glutamatergic responses in CeA neurons, a crucial substrate mediating the action of etha- 
nol, to our knowledge have not been investigated. Therefore, we have now studied the effects of acute and chronic ethanol on glutamatergic transmission within the CeA in a slice preparation and in vivo. In slices taken from rats that received chronic ethanol treatment (CET), acute ethanol elicited a greater inhibition of NMDAR-mediated responses than in naive rats and reduced paired pulse facilitation (PPF), an indicator of increased glutamate release. We extended these in vitro findings by demonstrating that in vivo administration of ethanol increases glutamate dialysate content in the CeA only after CET, supporting a presynaptic ethanol site of action. Our results indicate that CET induces neuroadaptative changes in CeA glutamatergic synapses that may be involved in behavioral signs of ethanol dependence.

\section{Materials and Methods}

Slice preparation. We prepared amygdala slices as previously described (Roberto et al., 2003) from male Sprague Dawley rats (120-300 gm) that were anesthetized with halothane (3\%) and decapitated. The brains were rapidly removed into ice-cold artificial CSF (ACSF) gassed with $95 \% \mathrm{O}_{2}$ and $5 \% \mathrm{CO}_{2}$. Transverse slices $400 \mu \mathrm{m}$ thick were cut on a Vibratome Series 3000 (Technical Products International, St. Louis, MO), incubated in an interface configuration for $\sim 30 \mathrm{~min}$, and then completely submerged and continuously superfused (flow rate of $2-4 \mathrm{ml} / \mathrm{min}$ ) with warm $\left(31^{\circ} \mathrm{C}\right.$ ), gassed ACSF of the following composition (in $\mathrm{mm}$ ): $\mathrm{NaCl}$, 130; $\mathrm{KCl}, 3.5 ; \mathrm{NaH}_{2} \mathrm{PO}_{4}, 1.25 ; \mathrm{MgSO}_{4} \cdot 7 \mathrm{H}_{2} \mathrm{O}, 1.5 ; \mathrm{CaCl}_{2}, 2.0 ; \mathrm{NaHCO}_{3}$, 24; and glucose, 10. In studies of the NMDAR-mediated responses, we used a modified ACSF containing reduced $\mathrm{Mg}^{2+}$ levels $(0.75 \mathrm{~mm})$. The inner chamber had a total volume of $0.8 \mathrm{ml}$; at the $2-4 \mathrm{ml} / \mathrm{min}$ superfusion rates used, $90 \%$ replacement of the chamber solution could be obtained within $1 \mathrm{~min}$. Drugs were added to the ACSF from stock solutions to obtain known concentrations.

CET. We used the standard ethanol inhalation method of The Scripps Research Institute Alcohol Research Center for developing CET rats (Rogers et al., 1979). This method has been used for $>25$ years, and all procedures are conducted in accordance with the National Institutes of Health Guide for the Care and Use of Laboratory Animals (Rogers et al., 1979). Briefly, male Sprague Dawley rats were housed two to four per cage with a 6 A.M.-6 P.M. light cycle and with ad libitum access to food and water. The animals were randomly divided into two groups and placed into either ethanol vapor chambers or into air-only chambers (sham controls). In the ethanol-treated group, we exposed rats to continuous ethanol vapors for at least 2 weeks. The health of the animals, weight gain, food and water intake, body posture, and locomotor activity were monitored daily. Sham controls were treated similarly but without exposure to ethanol vapor. On experiment days, animals in the CET group were maintained in vapor chambers until preparation of the amygdala slices. We made recordings in ethanol-free ACSF from slices of CET and sham rats $2-8 \mathrm{hr}$ after cutting the slices, corresponding to the period for the hyperexcitable behavioral response to ethanol withdrawal characteristic of dependence. Because we found no statistically significant $(p>0.1)$ differences in the basic electrophysiological measures or ethanol responses between neurons from naive control and sham control rats, we therefore pooled measures from these two groups.

Blood alcohol level and body weight. We determined blood alcohol levels (BALs) of the CET animals from tail blood samples taken three times/week. Control animals were also routinely bled. When necessary, we adjusted the ethanol vapor concentration after BAL measurement to achieve a constant target BAL of $150-200 \mathrm{mg} / \mathrm{dl}$. The mean BAL of all CET animals was $185.4 \mathrm{mg} / \mathrm{dl}(n=40)$. The CET animals did not show signs of deteriorating health, impairment of locomotor activity, or abnormality in body posture and gait. The mean body weight of CET animals was $250 \mathrm{gm}(n=23)$, compared with a mean body weight of $270 \mathrm{gm}$ $(n=10)$ for sham control animals. The mean body weights of 1 and 2 week withdrawn animals were $290(n=9)$ and $319(n=5) \mathrm{gm}$, respectively.

Electrophysiology. We recorded from CeA neurons with sharp micropipettes $(3 \mathrm{M} \mathrm{KCl})$ using the discontinuous voltage- or current-clamp mode. In the voltage-clamp mode, we used a switching frequency of 3-5 $\mathrm{kHz}$ and continuously monitored, on a separate oscilloscope, electrode settling time and capacitance neutralization at the headstage. The data were acquired with an Axoclamp-2A preamplifier (Axon Instruments, Foster City, CA) and stored for later analysis using pClamp software (Axon Instruments).

We evoked pharmacologically isolated EPSPs and EPSCs by stimulating locally within the CeA through a bipolar stimulating electrode. We pharmacologically isolated evoked AMPA receptor-mediated EPSPs and EPSCs by adding $30 \mu \mathrm{m}$ bicuculline (to block $\mathrm{GABA}_{\mathrm{A}}$ receptors), $1 \mu \mathrm{M}$ [1-(S)-3,4-dichlorophenyl)ethyl] amino-2-(S)-hydroxypropyl-p-benzylphosphonic acid (CGP 55845A) (to block $\mathrm{GABA}_{\mathrm{B}}$ receptors), and $30 \mu \mathrm{M}$ DL-2-amino-5-phosphonovalerate (D-AP-5; to block NMDARs) to the ACSF. We isolated NMDAR-mediated EPSPs and EPSCs in low- $\mathrm{Mg}^{2+}$ ACSF (0.75 instead of $1.5 \mathrm{~mm}$ ) using $30 \mu \mathrm{M}$ bicuculline, $1 \mu \mathrm{M}$ CGP 55845A, and $10 \mu \mathrm{M}$ 6-cyano-7-nitroquinoxaline-2,3-dione (CNQX; to block AMPA and kainate receptors).

In most neurons, we held the cells near their resting membrane potential (RMP; $-76 \mathrm{mV}$ ) and applied hyperpolarizing and depolarizing current steps (200 pA increments, 750 msec duration) to generate $V-I$ curves. We examined PPF in each neuron using 50, 100, and $180 \mathrm{msec}$ interpulse intervals. The stimulus strength was adjusted such that the amplitude of the first EPSP or EPSC was $50 \%$ of the maximal amplitude determined in an input-output (I-O) relationship. We calculated the PPF ratio as the second EPSP or EPSC amplitude over that of the first EPSP or EPSC. We took all measures before ethanol superfusion (control), during ethanol (5-10 $\mathrm{min})$, and after ethanol washout (20-30 min). We express all values as mean \pm SEM. We subjected data to a between-subjects or within-subject ANOVA with repeated measures and to the Newman-Keuls post hoc test, with $p<0.05$ considered statistically significant. When appropriate, we used Student's paired or unpaired $t$ test.

NMDA pressure application. We applied NMDA (10 mM) locally near the recorded neuron by pressure from a pipette (tip diameter, $2-4 \mu \mathrm{m}$; pressure, 1-10 psi; duration, $0.5-3 \mathrm{sec}$ ). The NMDA currents were recorded in voltage-clamp mode in low- $\mathrm{Mg}^{2+} \mathrm{ACSF}$ and in the presence of the GABA receptor blockers CGP 55845A (1 $\mu \mathrm{M})$ and bicuculline (30 $\mu \mathrm{M})$ together with CNQX $(10 \mu \mathrm{M})$ and $1 \mu \mathrm{M}$ TTX (to minimize presynaptic effects). The neurons were held near their RMPs $(-76 \mathrm{mV})$. After stable NMDA responses were achieved, we took peak current measurements at several points before, during, and after ethanol application. We defined an ethanol decrease of NMDA responses as a $\geq 10 \%$ decrease in peak response.

Drugs. CGP 55845A was a gift from Norvartis Pharma. We purchased D-AP-5 and CNQX from Tocris Cookson (Holloway Road, MO), bicuculline and NMDA from Sigma (St. Louis, MO), TTX from Calbiochem (San Diego, CA), ifenprodil tartrate from Research Biochemicals (Natick, MA), and ethanol from Remet (La Mirada, CA). To avoid loss of ethanol by evaporation, the solutions were diluted in gassed ACSF from sealed stock solutions of reagent grade $95 \%$ ethyl alcohol in water immediately before administration.

Microdialysis procedures. We conducted all procedures in accordance with the National Institutes of Health Guide for the Care and Use of Laboratory Animals. Sprague Dawley rats (250-350 gm) were anesthetized with isofluorane $(1-2 \%)$ and stereotaxically implanted with a stainless steel microdialysis guide cannula (21 gauge; Plastics One, Roanoke, VA), which terminated at the dorsal surface of the medial CeA (anteroposterior, $-2.3 \mathrm{~mm}$; mediolateral, $+4.0 \mathrm{~mm}$; ventral, $-6.4 \mathrm{~mm}$, from dura; Paxinos and Watson, 1986). After a $7 \mathrm{~d}$ recovery period, the animals were lightly anesthetized (1-2\% isoflourane), and microdialysis probes ( $1 \mathrm{~mm}$ active length, $0.6 \mu \mathrm{l} / \mathrm{min}$ ACSF perfusate flow rate: for details, see Frantz et al., 2002) were inserted and secured to the guide cannulas. Rats regained consciousness within 5 min of probe insertion, and a $3 \mathrm{hr}$ postimplantation period was given before dialysate collection. We collected microdialysate samples at $10 \mathrm{~min}$ intervals during a $60 \mathrm{~min}$ baseline period and 30 min periods during which ACSF containing 0.1, 0.3 , and $1.0 \mathrm{~m}$ ethanol was perfused through the probe. A new $40 \mathrm{~min}$ baseline period was obtained between each ethanol pulse, during which ethanol-free ACSF was the perfusate. To avoid technical artifacts arising 
from switching the perfusate solutions, the first sample collected after each perfusate change was not included in the final analyses. On the basis of previous work characterizing the delivery of ethanol by reverse dialysis (Robinson et al., 2000; Gonzales et al., 2002), the efficiency of ethanol delivery in the present study was estimated to be $\sim 10 \%$. Dialysate samples were frozen at $-70^{\circ} \mathrm{C}$ until they were derivatized with a fluorescent probe (naphthalene-2,3-dicarboxaldehyde) and analyzed for amino acid content using capillary electrophoresis with laser-induced fluorescence detection (see below).

In the ethanol-treated group, as described above for slice experiments, rats were continuously exposed to ethanol vapors for at least 2 weeks before microdialysis experiments. The mean BAL of these CET animals was 165.8 $\mathrm{mg} / \mathrm{dl}(n=7)$. Sham controls were treated similarly but without ethanol vapors. We implanted the stainless steel microdialysis guide cannula $7 \mathrm{~d}$ before the beginning of the CET. On experiment days, animals in the CET group were maintained in the ethanol vapor chamber until the microdialysis probes were inserted and secured to the guide cannulas. We collected the dialysate sample $3 \mathrm{hr}$ after probe implantation.

Amino acid analysis. We determined microdialysate amino acid content using capillary electrophoresis with laser-induced fluorescence detection. Amino acid derivatization was achieved by mixing $5 \mu \mathrm{l}$ of microdialysate with $9 \mu \mathrm{l}$ of $40 \mathrm{~mm}$ borate buffer, $\mathrm{pH} 9.2$, containing $3.8 \mathrm{~mm} \mathrm{KCN}$ and $1 \mu \mathrm{l}$ of $5 \mathrm{~mm}$ naphthalene-2,3dicarboxaldehyde in $\mathrm{MeOH}$. This mixture was allowed to react at room temperature in the dark for $30 \mathrm{~min}$ before placing the samples in the refrigerated $\left(10^{\circ} \mathrm{C}\right)$ sample tray of the capillary electrophoresis instrument (Agilent Technologies, Wilmington, DE). Samples were subsequently loaded onto a $90 \mathrm{~cm}$ fused silica capillary (30 $\mu \mathrm{m}$ inner diameter; sample loading by 50 millibars of pressure for $10 \mathrm{sec}$ ), and the amino acids were separated using $+15 \mathrm{kV}$ and a background electrolyte solution consisting of $100 \mathrm{~mm}$ borate buffer, $\mathrm{pH} 9.2$, containing $30 \mathrm{~mm}$ SDS and $2 \mathrm{~mm}$ hydroxypropyl- $\beta$ cyclodextrin. We detected the amino acids using a laser-induced fluorescence detector (Zetalif; Picometrics, Ramon Ville, France) with a $442 \mathrm{~nm} \mathrm{HeCd}$ laser $(30 \mathrm{~mW}$; Melles Griot,

Carlsbad, CA). External calibration standards were run in duplicate and were interspersed throughout the sample run. The limits of quantization were $\sim 1$ nM for each of the analytes. All reagents and amino acid standards were from Sigma.

\section{Results}

\section{Acute ethanol inhibits glutamatergic EPSPs and EPSCs}

We recorded from $141 \mathrm{CeA}$ neurons with a mean RMP of $-76 \pm$ $2 \mathrm{mV}$ and a mean input resistance of $107 \pm 5 \mathrm{M} \Omega$. Local electrical stimulation within the CeA evoked multicomponent postsynaptic responses consisting of glutamatergic EPSPs or EPSCs and GABAergic IPSPs or ISPCs. At RMP, the evoked responses were composed mainly of non-NMDA glutamate (AMPA and kainate) and $\mathrm{GABA}_{\mathbf{A}}$ receptor-mediated synaptic components. Single stimuli did not evoke additional components such as glycinergic, nicotinergic (Nose et al., 1991), or GABA $_{C}$-like (Delaney and Sah, 1999) responses. $15-26 \%$.
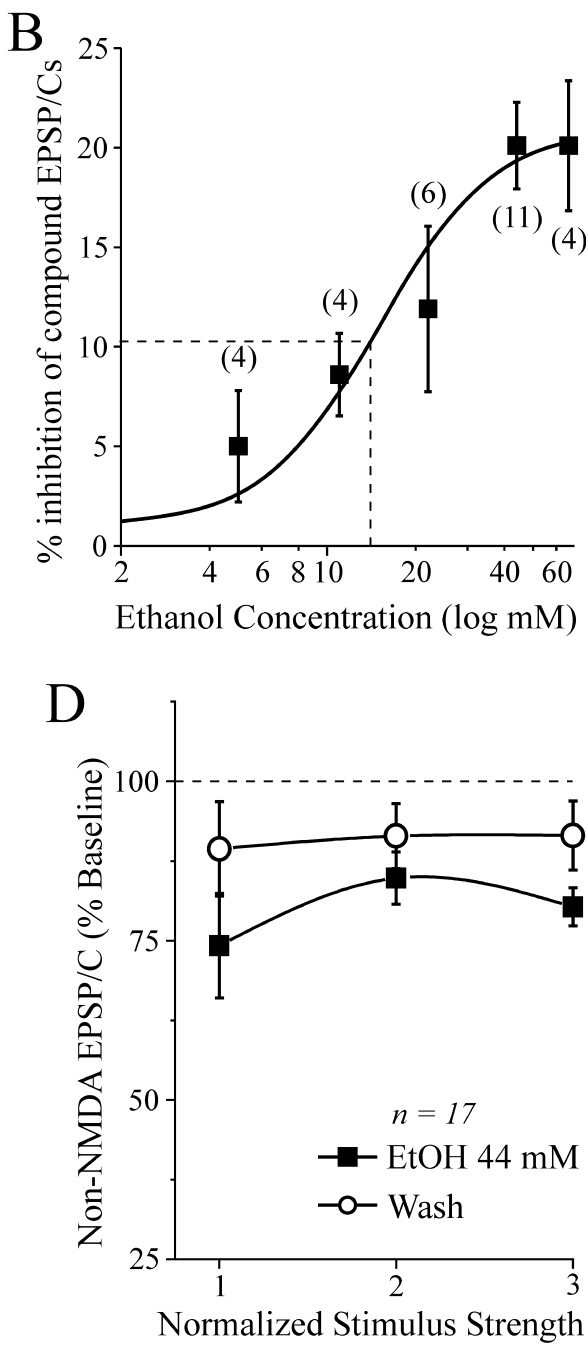

Figure 1. Acute ethanol decreases excitatory synaptic transmission in CeA neurons. $A$, Superfusion of 44 mm ethanol decreased the amplitude of evoked compound glutamatergic EPSCs isolated by GABAergic blockers. $B$, Concentration-response relationship 作 number of cells in parentheses). The logistic curve is plotted by Origin Software (Microcal) using $y=(A 1-A 2) /[\mathrm{sqb}] 1+(x / x 0) *$ 2]. Parameters of the logistic curve were set at upper asymptote fixed at $21 \%$ and lower at $1 \%$. The half-maximal inhibition EPSP and EPSC amplitudes elicited by $44 \mathrm{~mm}$ ethanol, averaged from 11 neurons. The mean EPSPs and EPSC s were significantly $(p<0.05)$ decreased by $26-39 \%$. D, Pooled data from 17 neurons; in the presence of $30 \mu \mathrm{m}$ bicuculline, $1 \mu \mathrm{m}$ CGP 55845A, and $20 \mu \mathrm{M}$ D-AP-5, $44 \mathrm{~mm}$ ethanol significantly $(p<0.05)$ decreased the non-NMDAR-mediated EPSP and EPSC amplitudes by

In a subset of neurons, we evoked compound glutamatergic EPSPs or EPSCs isolated by bicuculline and CGP 55845A. A dose-response analysis showed that ethanol (5-66 mM) superfusion significantly decreased the EPSP and EPSC amplitudes (Fig. $1 A$ ) across the range of stimulus intensities. The highest ethanol concentrations (44 and $66 \mathrm{~mm}$ ) produced a maximal inhibition of $21 \%$, with a half-maximal effect obtained at $14 \mathrm{~mm}$ (Fig. $1 B$ ).

Ethanol at $44 \mathrm{~mm}$ superfused for 7-10 min significantly $(p<$ $0.05 ; n=11$ ) decreased the amplitude of these EPSPs and EPSCs to $71.9 \pm 4 \%$ of control (measured at half-maximal stimulus intensity), with partial recovery on ethanol washout ( $84 \pm 9 \%$ of control; Fig. $1 A, C)$. Ethanol had no significant $(p>0.1)$ effect on basic membrane properties such as membrane potential, input resistance, or spike amplitude (data not shown), in agreement with our previous findings (Roberto et al., 2003).

The ethanol effect on these excitatory synaptic responses ap- 
A
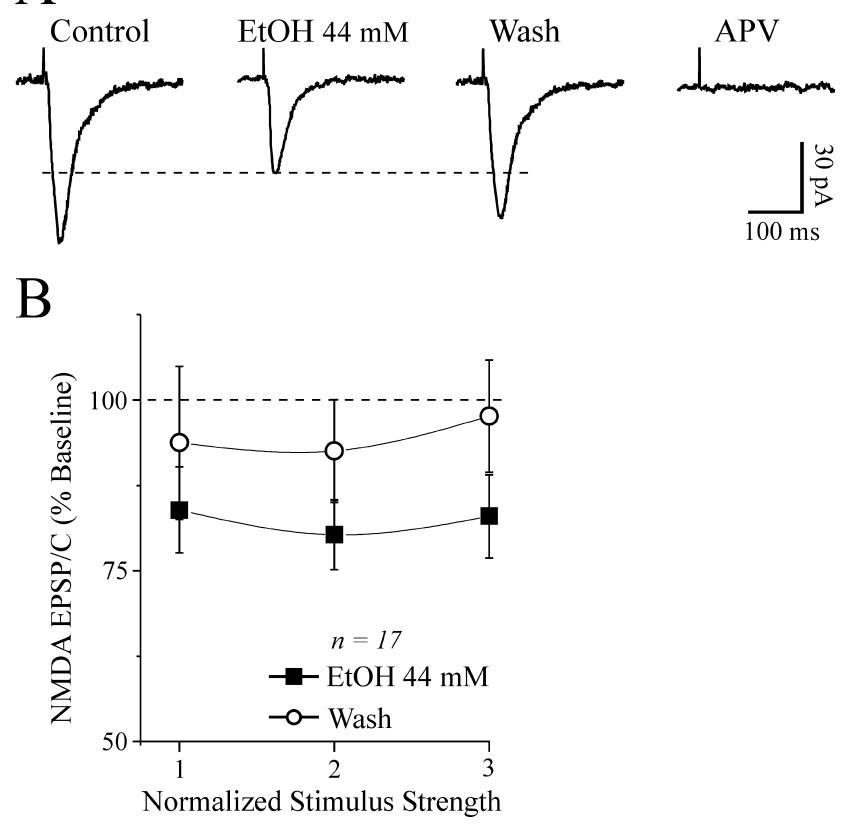

Figure 2. Acute ethanol decreases the amplitude of evoked NMDAR-mediated EPSPs and EPSCS. A, Representative NMDAR-mediated EPSCs evoked in the presence of $30 \mu \mathrm{m}$ bicuculline, $1 \mu \mathrm{M} \mathrm{CGP55845A,} 10 \mu \mathrm{M}$ CNQX, and low $\mathrm{Mg}^{2+}$ concentrations. Ethanol decreased the NMDARmediated EPSC amplitudes with partial recovery on washout. The subsequent addition of D-AP-5 completely blocked the EPSCs, implicating NMDARs. $B$, In slices from naive rats, superfusion of 44 mm ethanol significantly ( $p<0.01 ; n=17$ ) reduced evoked NMDAR-mediated EPSPs and EPSCs by $15-20 \%$.

peared to be mediated primarily by a reduction of the nonNMDAR-mediated component. In fact, D-AP-5 (10 $\mu \mathrm{M})$ did not significantly mitigate the inhibitory effect of ethanol on EPSPs and EPSCs, and in most neurons, the addition of $10 \mu \mathrm{M}$ CNQX totally blocked the EPSPs and EPSCs. Thus, in normal ACSF and at RMP, the evoked EPSPs and EPSCs are composed of nonNMDA glutamatergic components. In a subset of experiments ( $n=4$ ) without CGP 55845A (but still with bicuculline), there was no quantitative or qualitative difference in the ethanolinduced inhibition of glutamatergic responses under theses conditions (data not shown). Thus, as in our previous report showing ethanol augmentation of both GABA IPSPs and GABA currents in CeA in absence of a $\mathrm{GABA}_{\mathrm{B}}$ receptor antagonist (cf. Wan et al., 1996 vs Roberto et al., 2003), $\mathrm{GABA}_{\mathrm{B}}$ receptors do not appear to play a role in the ethanol inhibition of EPSP and EPSC amplitudes.

We studied the isolated non-NMDAR-mediated component in 17 neurons held at RMP $(-77 \pm 1 \mathrm{mV})$. In the presence of D-AP-5, CGP 55845A, and bicuculline, ethanol superfusion (44 $\mathrm{mM}, 7-10 \mathrm{~min})$ significantly decreased the non-NMDARmediated EPSP and EPSC amplitudes by $15-26 \%$ across the stimulus strengths used, with recovery on washout (Fig. $1 D$ ). The I-O curves of the compound glutamatergic EPSPs and EPSCs and non-NMDAR-mediated EPSPs and EPSCs measured before application of ethanol were comparable $(p>0.05$; data not shown), again indicating that in this experimental condition, the NMDAR-mediated component at CeA synapses is negligible. However, we could evoke D-AP-5-sensitive, voltage-dependent NMDAR-mediated EPSPs and EPSCs by using a low- $\mathrm{Mg}^{2+}$ ACSF. Acute ethanol (44 mm) also decreased these pharmacologically isolated NMDAR-mediated EPSPs and EPSCs by $15-20 \%$ $(n=17 ; p<0.01)$ over the stimulus strengths used (Fig. $2 A, B)$.

\section{Effects of chronic ethanol on evoked glutamatergic EPSPs and EPSCs}

Because glutamate receptors, and especially NMDARs, are thought to be involved in ethanol-related phenomena such as tolerance, dependence, and withdrawal, we recorded from slices taken from rats chronically exposed to ethanol vapors for 2-4 weeks. We prepared the CeA slices from CET rats exactly as for the acute ethanol experiments and allowed the slices to withdraw from ethanol in the recording chamber.

To determine whether the CET animals were ethanoldependent, at the end of the CET period, we assessed the performance of a separate sample of CET rats on several behavioral tests for ethanol withdrawal severity. Withdrawal signs, evaluated by a common behavioral rating scale for hyperactivity (ventromedial distal limb flexion response, tail stiffness, and abnormal body posture and gait; Macey et al., 1996), were measured at 2, 4, 6, and $8 \mathrm{hr}$ after ethanol withdrawal in the CET group and compared with similar measures made in the naive group. Behavioral signs of ethanol withdrawal were evident in all six rats tested at $2-8 \mathrm{hr}$ after the termination of the CET, a time frame corresponding to the electrophysiological recordings.

However, we did not observe signs of postsynaptic neuronal hyperexcitability in CeA slices, such as prolonged depolarizations or spontaneous burst discharges, after withdrawal. In fact, we did not observe a significant difference between the baseline compound EPSP and EPSC I-O curves in slices from naive and CET animals (data not shown). The baseline I-O curves for NMDARmediated EPSPs and EPSCs were also comparable in naive and CET rats (data not shown). However, baseline I-O curves for the non-NMDAR-mediated EPSPs and EPSCs were significantly lower at the highest stimulus intensities in slices from CET rats compared with slices from naive and sham rats (Fig. $3 A$ ). In slices taken from CET rats after early withdrawal $(2-8 \mathrm{hr}$ after ethanol exposure), superfusion of $44 \mathrm{~mm}$ ethanol significantly $(p<0.01)$ decreased the evoked compound and non-NMDAR-mediated EPSPs and EPSCs (Fig. 3B,C) by $20 \pm 6$ and $19 \pm 7 \%$ of control, respectively, similar to the ethanol effect in slices taken from naive rats (compare Fig. $1 C, D$ ).

By contrast, the depressant effect of acute $44 \mathrm{~mm}$ ethanol on NMDA-EPSP and EPSC amplitudes was significantly $(p<0.05)$ stronger in slices from CET rats (EPSPs and EPSCs reduced by 25-40\%; Fig. 4A) than in those from naive rats (EPSPs and EPSCs reduced by 15-20\%; Fig. 2). Ethanol at $44 \mathrm{~mm}$ significantly $(p<0.05)$ decreased the amplitude of NMDAR-mediated EPSPs in eight neurons from CET rats, with only partial recovery on washout (Fig. $4 \mathrm{~B}$ ). Although the ethanol inhibition of NMDARmediated EPSPs was greater in neurons from CET rats compared with naive rats, the washout (recovery) values also were depressed (Fig. 4A,B). Therefore, we performed further experiments to determine whether the ethanol effect on NMDARmediated EPSPs and EPSCs was ethanol-specific and not attributable to NMDAR and channel rundown in CET rats. First we recorded NMDAR-mediated EPSPs or EPSCs in neurons taken from CET rats and superfused with ACSF without ethanol for $>45$ min (Fig. 4C). We observed that the NMDAR-mediated EPSP and EPSC amplitudes remained unaffected over such recording periods in both CET rats $(n=6$; Fig. $4 C)$ and naive rats ( $n=3$; data not shown). We then compared the effect of competitive (D-AP-5) and noncompetitive (ifenprodil) NMDAR antagonists on the ethanol-induced inhibition of NMDARmediated EPSPs and EPSCs. Ifenprodil provides near-maximal block of NMDARs containing the NR2B subunit (Williams, 2001), which is strongly implicated in ethanol effects. In low- 
$\mathrm{Mg}^{2+}$ ACSF containing GABA receptor blockers and CNQX, D-AP-5 (10 $\mu \mathrm{M})$ completely blocked NMDAR-mediated EPSPs and EPSCs ( $n=6$; data not shown). Ifenprodil $(10 \mu \mathrm{M})$ had a small effect in neurons of naive rats $(5-15 \% ; n=6)$, but significantly reduced the NMDARmediated EPSP and EPSC amplitudes by $20-30 \%$ in neurons of CET rats $(p<0.05$ compared with baseline or ifenprodil in naive slices; $n=5$; Fig. 5). Interestingly, superfusion of $44 \mathrm{~mm}$ ethanol in the presence of ifenprodil did not further depress NMDAR-mediated EPSPs and EPSCs.

\section{Effects of acute and chronic ethanol on PPF}

To determine whether the inhibitory effect of ethanol on glutamatergic transmission involved presynaptic changes in glutamate release, we examined PPF $(50,100$, and $180 \mathrm{msec}$ interpulse intervals) of compound and non-NMDAR- and NMDARmediated EPSPs and EPSCs in CeA neurons before, during, and after ethanol application. Changes in PPF are thought to be inversely related to changes in transmitter release such that enhanced probability of release is associated with a reduction of PPF (Mennerick and Zorumski, 1995; Bonci and Williams, 1997). We found that baseline PPF of non-NMDARand NMDAR-mediated EPSPs and EPSCs in neurons from naive and CET animals was similar at all interpulse intervals tested (Fig. 6B). Also, superfusion of $44 \mathrm{~mm}$ ethanol onto control slices had little effect on PPF of NMDAR-mediated EPSPs and EPSCs (Fig. 6C). By contrast, acute $44 \mathrm{~mm}$ ethanol significantly $(p<0.05)$ decreased PPF of NMDAR-mediated EPSPs and EPSCs by $15 \%$ in slices from CET rats $(n=$ 16; Fig. $6 A, C)$, suggesting an acute ethanol-induced increase in glutamate release and a neuroadaptative change after CET. Acute ethanol had equivalent effects on PPF of both compound and nonNMDAR-mediated EPSPs and EPSCs; that is, there was no acute ethanol effect on PPF in naive rats, but PPF was decreased by $15-22 \%$ in CET rats (data not shown). This effect returned to control levels after 2 weeks of withdrawal (our unpublished observations).

\section{Effects of acute and chronic ethanol on postsynaptic NMDARs}

Because acute ethanol reduced NMDARmediated EPSP and EPSC amplitudes with little effect on PPF in neurons of naive rats, we hypothesized that the ethanol effect may take place at postsynaptic sites, perhaps on the NMDAR itself. To test this hy-

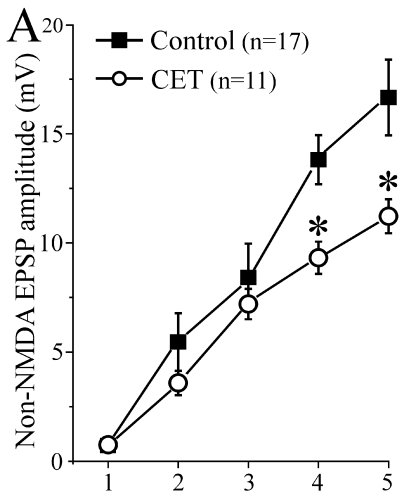

Normalized Stimulus Strength

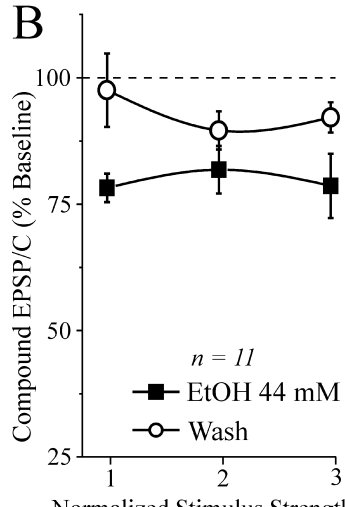

Normalized Stimulus Strength

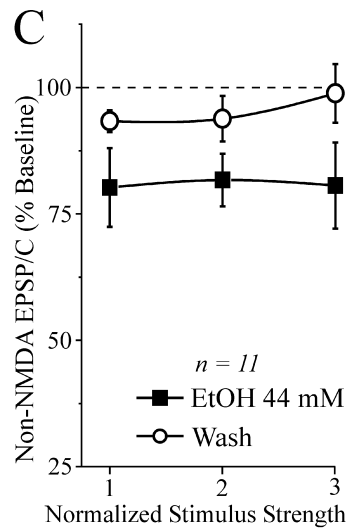

Figure 3. CET reduces baseline non-NMDA transmission but does not alter acute ethanol effects. $A, I-0$ curves of non-NMDARmediated EPSP and EPSC amplitudes evoked by local stimulation. At higher stimulus strengths, the baseline response is significantly $\left({ }^{*} p<0.05\right)$ reduced in slices taken from CET rats compared with controls. B, Acute ethanol significantly $(p<0.05)$ decreased by $20 \%$ compound EPSPs and EPSCs in slices from CET rats, to a similar extent as those from control slices. $C$, In neurons ofCET rats, acute ethanol significantly $(p<0.05 ; n=11$ ) reduced non-NMDAR-mediated EPSPs and EPSCs (with partial recovery on washout) to a similar extent as in control slices.
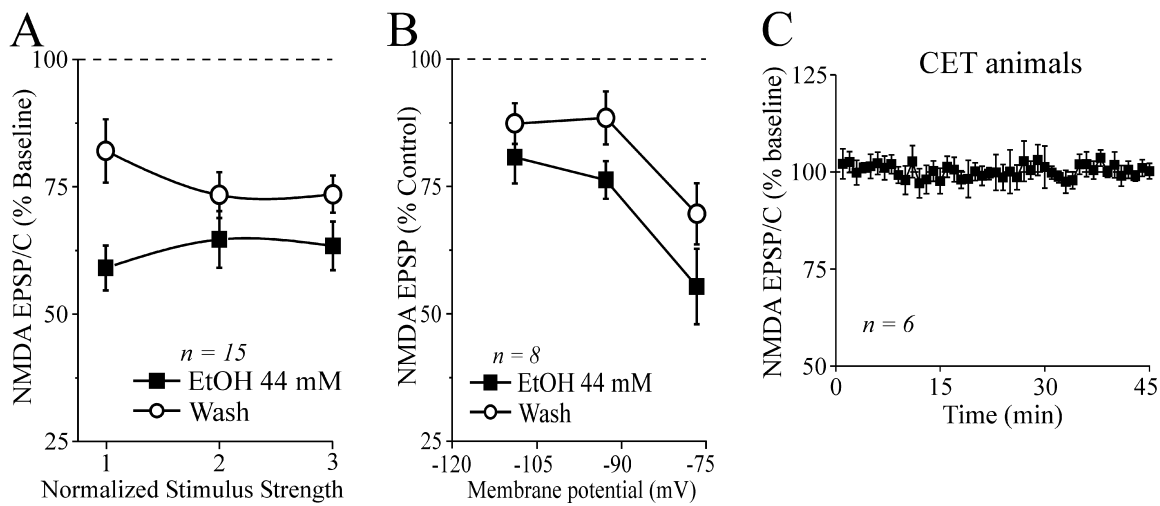

Figure 4. CET potentiates the inhibitory effect of acute ethanol on NMDAR-mediated EPSPs and EPSC $\operatorname{in} C$ CA. A, Acute ethanol reduced NMDAR-mediated EPSP and EPSC amplitudes by $35-45 \%$ in neurons from CET rats $(n=15)$ compared with $15-20 \%$ in neurons from naive rats (Fig. $2 B$ ), suggesting sensitization to acute ethanol. $B$, Ethanol superfusion significantly $(p<0.001 ; n=$ 8) decreased evoked NMDAR-mediated EPSP amplitudes across the voltage range tested (with only partial recovery on washout), especially at the more depolarized potentials. C, In neurons from CET rats, evoked NMDAR-mediated EPSPs and EPSCs remained stable for $>45$ min of superfusion in the absence of ethanol.
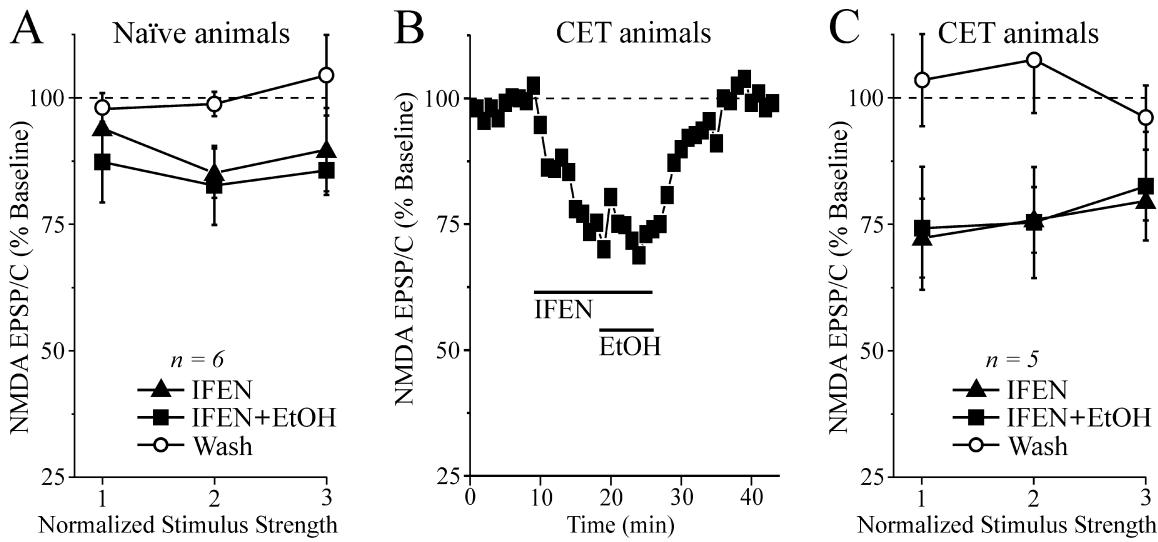

Figure 5. The NR2B blocker ifenprodil occludes the depressant effect of acute ethanol. $A$, Bath application of $10 \mu \mathrm{m}$ ifenprodil (IFEN) for 10 min decreased the NMDAR-mediated EPSP and EPSC amplitudes $(n=6)$. Subsequent superfusion of 44 mm ethanol (EtOH) did not further affect the NMDAR-mediated EPSPs and EPSCs in these neurons taken from naive rats. $B$, Time course record depicting the sequential application of $10 \mu \mathrm{m}$ ifenprodil and $44 \mathrm{~mm}$ ethanol on NMDAR-mediated EPSC amplitudes in a neuron from a CET rat. C, Summary of the effect of ifenprodil and ethanol on five CeA neurons from CET rats. Ifenprodil significantly ( $p<$ 0.05) decreased NMDAR-mediated EPSP and EPSC amplitudes and prevented the ethanol effect, with full recovery on drug washout. 


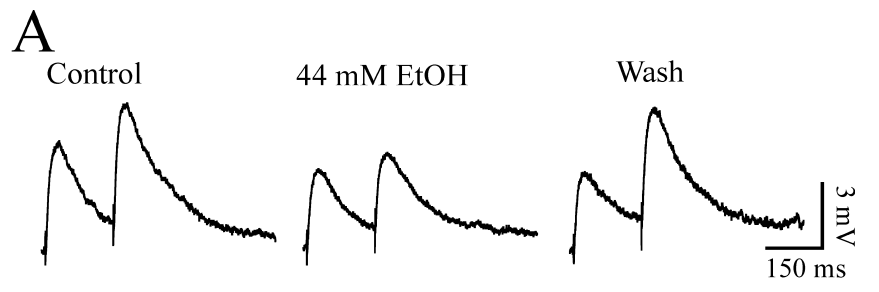

B

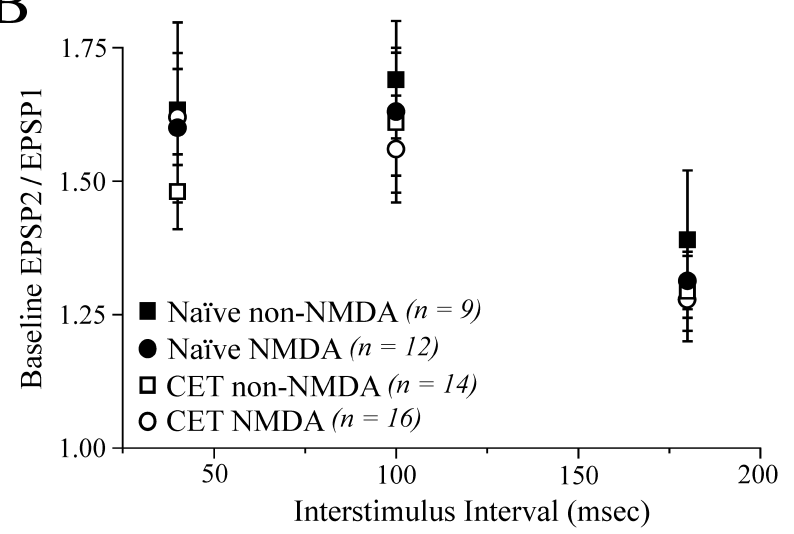

C

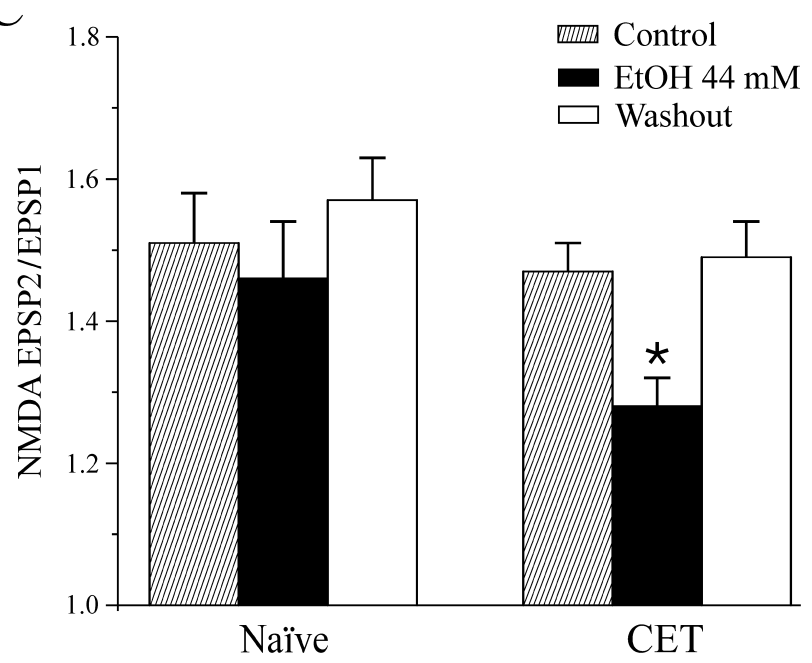

Figure 6. Baseline PPF is unchanged after CET, and acute ethanol decreases PPF of NMDARmediated EPSPs and EPSCs but only after CET. $A$, Voltage records of NMDAR-mediated EPSPs in response to two stimuli $100 \mathrm{msec}$ apart in a neuron from a CET rat. Acute ethanol reduced PPF. $B$, Pooled data of baseline PPF ratios (at 50,100, and 180 msec interpulse intervals) of nonNMDAR- and NMDAR-mediated EPSPs and EPSCs in neurons of naive and CET rats. There was no significant difference between groups. C, Pooled data of PPF ratio expressed as the second EPSP or EPSC amplitude over the firstEPSP or EPSC. Superfusion of ethanol significantly decreased the PPF ratio in neurons from CET (by $15 \%$; $\left.^{*} p<0.05 ; n=16\right)$ but not naive $(n=10)$ rats.

pothesis, we studied the effects of acute and chronic ethanol on responses to NMDA (10 mM) locally applied by pressure in the presence of TTX to minimize presynaptic effects. We superfused ethanol (44 mM) after a stable baseline of NMDA responses had been obtained (10-15 min). As predicted from the NMDARmediated EPSP and EPSC data and in accord with past studies showing ethanol inhibition of NMDA responses in other neuron types (Hoffman et al., 1989; Lovinger et al., 1989; Simson et al., 1993; Nie et al., 1994), acute ethanol decreased currents elicited by exogenous NMDA to $70 \pm 5 \%$ of baseline $(p<0.05 ; n=4$; Fig. 7) with recovery on washout. In addition, as with the NMDAR-mediated EPSPs and EPSCs, ethanol superfusion elicited a significantly greater inhibition (to $47 \pm 7 \%$; $p<0.001$
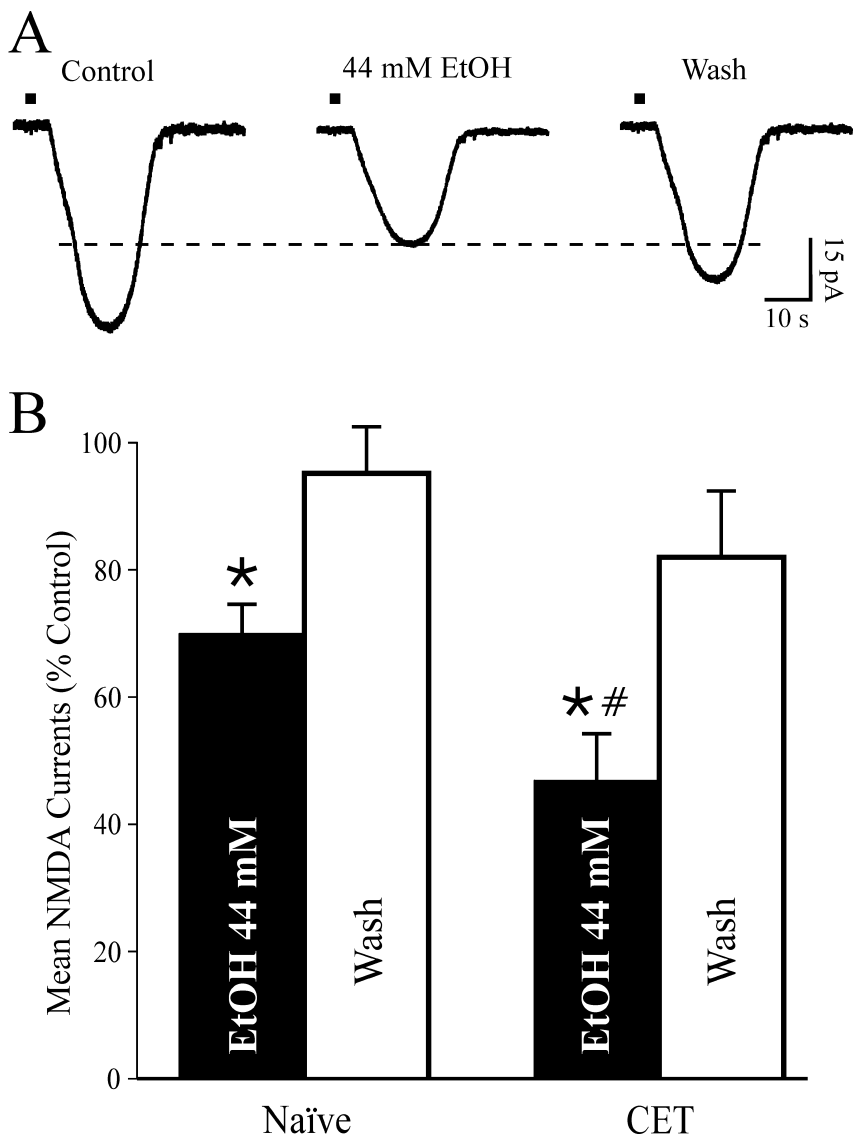

Figure 7. Inward currents elicited by local application of NMDA are decreased by acute ethanol. $A$, Representative current records before, during, and after ethanol superfusion ( $8 \mathrm{~min}$ ) from a neuron of a CET rat. Local pressure application ( $2 \mathrm{sec}, 6 \mathrm{psi}$; bars above the records) of NMDA (10 mm in the pipette, applied every $2 \mathrm{~min}$ ) in low- $\mathrm{Mg}^{2+}$ ACSF containing GABAergic blockers CNQX and $1 \mu \mathrm{M}$ TTX. $B$, In 4 neurons from naive rats, superfusion of $44 \mathrm{~mm}$ ethanol (5-10 min) significantly $\left({ }^{*} p<0.05\right)$ decreased exogenous NMDA-induced currents by $30 \pm$ $5 \%$, with recovery on washout ( $95 \pm 7 \%$ of control, $10-15 \mathrm{~min}$ ). In four neurons from CET rats, $44 \mathrm{~mm}$ ethanol more strongly decreased NMDA currents by $53 \pm 7 \%$ ( ${ }^{*} p<0.001$ compared with baseline; ${ }^{*} p<0.05$ compared with ethanol-induced inhibition in control slices) with partial recovery on washout (to $82 \pm 10 \%$ of control).

compared with baseline; $p<0.05$ compared with ethanolinduced inhibition in naive rats; $n=4)$ of the NMDA currents evoked in slices taken from CET rats (Fig. 7), again suggesting a sensitization or reverse tolerance but now at the postsynaptic level. As with the evoked NMDAR-mediated EPSPs and EPSCs, the depressant effect of acute $44 \mathrm{~mm}$ ethanol on exogenous NMDA-induced currents did not totally recover (to $82 \pm 10 \%$ of control; $n=3$; 15-20 min of washout; Fig. 7) during or after washout of ethanol.

\section{Ethanol microdialysis: in vivo glutamate content}

To directly determine whether ethanol alters glutamate release in $\mathrm{CeA}$, we measured the effects of local application of ethanol dialysate on glutamate levels in CeA in vivo. In sham control rats, the baseline concentration of dialysate glutamate was $1.2 \pm 0.2 \mu \mathrm{M}$ $(n=11$; Fig. $8 B$ ). This concentration profile agrees with a previous report of amygdala dialysate levels (Quertemont et al., 1998). In sham control rats, perfusion of the microdialysis probe with $0.1,0.3$, and $1.0 \mathrm{M}$ ethanol into the CeA did not significantly $(p>$ 0.05 ) alter the local dialysate glutamate levels (increase compared with baseline, $114 \pm 72,43 \pm 110$, and $153 \pm 139 \mathrm{~nm}$, respectively; Fig. $8 C$ ). In contrast, in CET rats, the baseline glutamate 

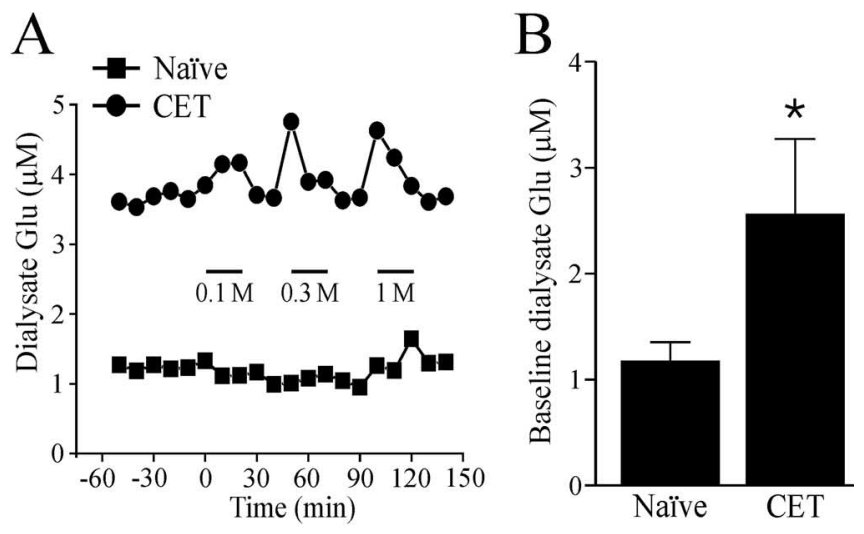

$\mathrm{C}$

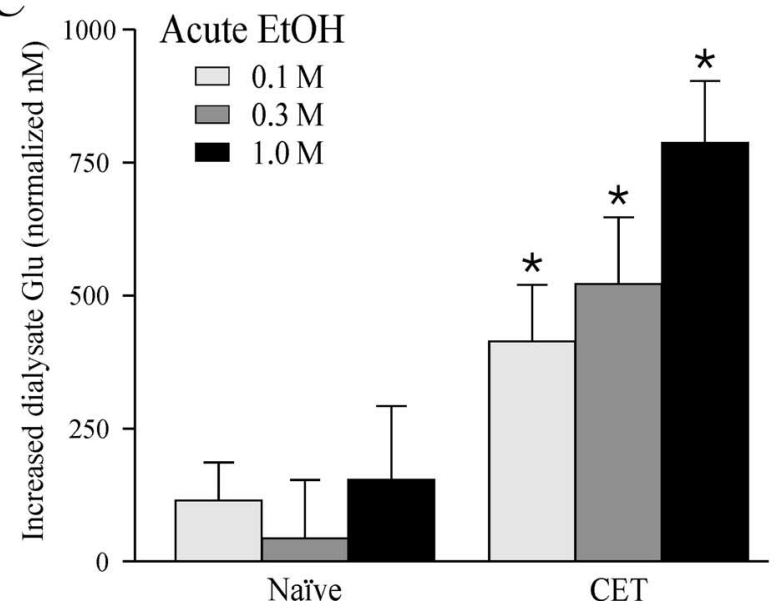

Figure 8. In vivo dialysate levels of glutamate in CeA. A, Representative samples of dialysate glutamate levels from naive (squares) and CET (circles) rats depicting the effect of ethanol administration (0.1, 0.3, and $1.0 \mathrm{~m}$; bars). $B$, In CET rats, the baseline dialysate glutamate level was increased $(2.6 \pm 0.8 \mu \mathrm{m} ; n=7)$ compared with that in naive rats $\left(1.2 \pm 0.2 \mu \mathrm{m} ;{ }^{*} p<\right.$ $0.05 ; n=11) . C$, In naive rats, local in vivo ethanol administration $(0.1,0.3$, and $1.0 \mathrm{M})$ into the CeA did not significantly ( $p>0.05$ ) alter local dialysate glutamate (Glu) levels (increase compared with baseline, $114 \pm 72,43 \pm 110$, and $153 \pm 139$, nm, respectively; $n=11$ ). In agreement with our in vitro electrophysiological results, only in CET rats did local infusion of ethanol produce a significant dose-dependent increase in dialysate glutamate levels (increase compared with baseline, $413.3 \pm 107.9,522 \pm 125$, and $787.4 \pm 116.0 \mathrm{~nm}$, respectively; $n=$ $\left.7 ;{ }^{*} p<0.05\right)$. Dialysates were normalized to baseline level.

microdialysate content was significantly $(p<0.05$; $n=7)$ increased $(2.6 \pm 0.8 \mu \mathrm{M})$ compared with that in naive rats $(1.2 \pm$ $0.2 \mu \mathrm{M}$; Fig. $8 B$ ). Furthermore, local administration of $0.1,0.3$, and 1.0 M ethanol also led to a significant $(p<0.05)$ dosedependent increase in dialysate glutamate levels (increase compared with baseline, 413.3 $\pm 107.9,522 \pm 125$, and 787.4 \pm 116.0 nm, respectively; Fig. $8 C$ ). A representative microdialysis time course record shows that in each case, dialysate glutamate levels returned to preethanol baseline levels when the perfusion medium was replaced with ethanol-free ACSF (Fig. 8A).

\section{Discussion}

We have shown that acute ethanol decreases both NMDAR- and non-NMDAR-mediated responses in CeA slices taken from both naive and CET rats. In slices from CET rats, acute ethanol superfusion selectively inhibited NMDAR-mediated EPSPs and EPSCs to a greater degree than in those from control rats, concomitant with a decreased PPF suggestive of an increase in glutamate release. Acute ethanol also elicited a more pronounced inhibition of the NMDA currents evoked by locally applied NMDA (in the presence of TTX) in slices taken from CET rats compared with those from naive rats, suggesting a sensitization or reverse tolerance to ethanol at the postsynaptic level. The inhibition of NMDAR-mediated EPSPs and EPSCs by the noncompetitive NR2B-specific antagonist ifenprodil also was increased in neurons from CET rats. Ifenprodil also completely occluded the ethanol inhibition of NMDAR-mediated EPSPs and EPSCs, suggesting that the ethanol effect was mediated by NR2B subunits. Using microdialysis, we found that in CET rats the intra-CeA administration of ethanol $(0.1-1.0 \mathrm{M})$ via reverse microdialysis significantly increased dialysate glutamate content compared with naive rats, supporting the PPF data suggesting a presynaptic action. Overall, our findings support the hypothesis that ethanol intoxication, reinforcement, or both may be associated with depression of glutamatergic transmission, whereas the dysregulation of glutamatergic synapses resulting from the combination of increased NMDAR sensitivity to ethanol and increased glutamate release may contribute to ethanol dependence.

A prominent electrophysiological effect of acute ethanol is a decrease in current generated by NMDAR activation (Kumari and Ticku, 2000; Woodward, 2000; Lovinger, 2002). The ability of ethanol to inhibit NMDARs may vary among brain regions (Yang et al., 1996; Kumari and Ticku, 2000; Criswell et al., 2003). Although the exact mechanisms underlying such variability remain to be elucidated at the molecular level, it is likely that they reflect differences in the various isoforms constituting the NMDA-receptor complex (Koltchine et al., 1993; Mirshahi and Woodward, 1995) as well as other neuron-specific factors (e.g., subunit phosphorylation) that may modulate the sensitivity of NMDAR channels to ethanol (Kuner et al., 1993; Masood et al., 1994). For example, the degree of ethanol inhibition may be dependent on the NR2 subunit expressed; NMDARs with NR1/2A and NR1/2B subunits may be the most sensitive to ethanol (Lovinger, 1995; Mirshahi and Woodward, 1995; Allgaier, 2002). Adaptations within the glutamatergic system have been suggested to contribute to ethanol tolerance and dependence and both acute and protracted features of ethanol withdrawal (Tabakoff and Hoffman, 1996; Tsai and Coyle, 1998; Krystal et al., 2003). CET alters the glutamatergic system in several brain regions, including hippocampus, cerebral cortex, striatum, and thalamus (Lovinger, 1997; Little, 1999; Kumari and Ticku, 2000; Dettmer et al., 2003; Kumari et al., 2003). However, the exact molecular mechanisms responsible for such effects after CET remain to be determined. CET increases the number of binding sites for several NMDAR ligands and alters NMDAR subunit expression (Trevisan et al., 1994; Kalluri et al., 1998; Kumari and Ticku, 2000), which could reflect an adaptive upregulation of NMDAR complexes in response to long-term ethanol exposure.

We recorded from CeA slices that were allowed to undergo withdrawal 2-8 hr after removal from CET, and we did not observe postsynaptic hyperexcitability. In these slices, the basal compound glutamatergic synaptic responses and the baseline PPF were comparable in CeA neurons from naive and CET rats. However, we found a significant difference between CeA neurons of these two groups in the I-O curves for the non-NMDARmediated EPSPs and EPSCs evoked over the highest range of stimulus strengths. Furthermore, acute superfusion of ethanol also decreased the amplitudes of both evoked NMDAR-mediated EPSPs and EPSCs and the responses to locally applied NMDA to a greater extent in CeA slices of CET rats than from naive rats, suggesting sensitization of NMDARs to ethanol after CET. This receptor sensitization could represent the cellular underpinnings for some aspects of behavioral sensitization seen in dependent 
animals (Xie et al., 1995; Darbra et al., 2002; Quadros et al., 2002; Broadbent et al., 2003; Meyer and Phillips, 2003). It has been reported that in the CeA the NMDARs are composed mainly of NR1 and NR2B subunits, and there is no developmental change in subunit composition (Lopez de Armentia and Sah, 2003; Sah and Lopez De Armentia, 2003). Interestingly, we found that the NR2B subunit-selective antagonist ifenprodil is more effective in inhibiting the NMDAR-mediated EPSPs and EPSCs and in occluding the ethanol effect in CET rats than in naive rats. These findings suggest that an increase in NR2B subunit composition may be involved in the acute effect of ethanol after CET.

There may be both direct (on NMDAR subunits) and indirect (on intracellular targets such as kinases and phosphatases) mechanisms that mediate ethanol effects on the NMDARs. We hypothesize that, in CeA, increased ethanol inhibition of NMDARs also could be attributable to changes in tyrosine phosphorylation of NR2B subunits. Potential mechanisms downstream of changes in phosphorylation include modulation of channel properties, regulation of subunit interactions, and altered receptor localization (e.g., greater surface expression of the receptors). It is also possible that the responsiveness of the trafficking function of the receptors to acute ethanol may increase during CET and may lead to alterations of internalization or of insertion of the NMDARs. Recent studies of NMDARs have focused on dynamic regulation of surface expression as a mechanism of ethanol action (Maldve et al., 2002; Alvestad et al., 2003; Yaka et al., 2003). These studies indicate that the inhibitory effect of ethanol on NMDARs involves a reduced tyrosine phosphorylation of NR2A and NR2B subunits, possibly via activation of a tyrosine phosphatase. Specifically, a Fyn kinase determines NMDAR sensitivity to ethanol (Yaka et al., 2003), and tyrosine phosphorylation of this Fyn kinase site is reduced by ethanol (Alvestad et al., 2003). Therefore, ethanol induces a phosphorylation-dependent enhancement of NMDAR channels. An alternative hypothesis involves the translocation of NMDARs between synaptic and extrasynaptic sites in response to ethanol. Molecular studies are now in progress in our laboratory to determine whether this sensitization is associated with changes in the phosphorylation status of the NR2Bcontaining NMDARs (nontranscriptional mechanisms), upregulation in NR2B mRNA transcription, or both.

A few reports have shown that ethanol decreases glutamate release in the hippocampus (Moghaddam and Bolinao, 1994; Reynolds and Brien, 1994; Gonzales and Brown, 1995; Tsai, 1998). Here we estimated the interstitial glutamate concentrations in the CeA using microdialysis in freely moving rats. In agreement with our in vitro electrophysiological results, the in vivo data showed that acute ethanol had no effect on glutamate release in naive rats but increased glutamate release in CET rats. We also found that baseline dialysate levels of glutamate in the CeA were elevated in dependent animals, suggesting that the increase in the glutamate content may be related to changes in presynaptic function after the CET and could represent a compensatory response to the continuous inhibitory effect (attributable to the reverse tolerance; see above) of ethanol on postsynaptic glutamate receptors. This enhancement in glutamate levels or release could also represent a response by the CeA to oppose the inhibitory effects of acute ethanol caused by the enhancement of GABAergic neurotransmission previously reported by our laboratory (Roberto et al., 2003). The increase in dialysate glutamate could represent synaptically released glutamate, carriermediated release, a change in glial metabolism, or a combination thereof (Timmerman and Westerink, 1997). Thus, part of the amino acid content of the dialysate may originate from non- impulse-dependent sources. Further studies (e.g., calcium removal or TTX inclusion) will ascertain whether the enhancement of glutamate dialysate is attributable to neuronal release, glutamate transporters, or both.

In conclusion, we have shown that acute and chronic ethanol significantly alters CeA glutamatergic transmission, involving both presynaptic and postsynaptic sites. Whereas acute ethanol appears to act predominantly at postsynaptic sites to decrease responses to glutamate, chronic ethanol causes an increased sensitivity to acute ethanol of postsynaptic NMDARs and increased presynaptic glutamatergic release. These changes with chronic ethanol may represent neuroadaptations or allostatic responses underlying behavioral signs of ethanol dependence (Koob and Le Moal, 2001). Although these two persistent effects might seem to counter each other in the presence of ethanol, they could bias the synapses toward hyperstimulation of non-NMDARs and reduced NMDAR function, resulting in altered spatial and temporal integrative properties of CeA neurons. The combination of these effects with enhanced GABAergic transmission by ethanol (Roberto et al., 2003) may tend to depress neuronal activity of CeA neurons, especially in the depolarized range. Further studies of ethanol effects on the integrative network and plasticity characteristics of the amygdala may help further elucidate the biological substrates of the reinforcing effects of ethanol consumption and how those effects change during the development of dependence.

Note added in proof. We overlooked a microdialysis study by Rossetti and Carboni (1995) showing increased glutamate levels in striatum 12-24 hr after withdrawal from systemic chronic ethanol treatment.

\section{References}

Allgaier C (2002) Ethanol sensitivity of NMDA receptors. Neurochem Int 41:377-382.

Alvestad RM, Grosshans DR, Coultrap SJ, Nakazawa T, Yamamoto T, Browning MD (2003) Tyrosine dephosphorylation and ethanol inhibition of N-Methyl-D-aspartate receptor function. J Biol Chem 278:11020-11025.

Bonci A, Williams JT (1997) Increased probability of GABA release during withdrawal from morphine. J Neurosci 17:796-803.

Broadbent J, Kampmueller KM, Koonse SA (2003) Expression of behavioral sensitization to ethanol by DBA/2J mice: the role of NMDA and nonNMDA glutamate receptors. Psychopharmacology 167:225-234.

Calton JL, Wilson WA, Moore SD (1998) Magnesium-dependent inhibition of $N$-methyl-D-aspartate receptor-mediated synaptic transmission by ethanol. J Pharmacol Exp Ther 287:1015-1019.

Criswell HE, Ming Z, Griffith BL, Breese GR (2003) Comparison of effect of ethanol on $N$-methyl-D-aspartate- and GABA-gated currents from acutely dissociated neurons: absence of regional differences in sensitivity to ethanol. J Pharmacol Exp Ther 304:192-199.

Darbra S, Prat G, Pallares M, Ferre N (2002) Tolerance and sensitization to the hypnotic effects of alcohol induced by chronic voluntary alcohol intake in rats. J Psychopharmacol 16:79-83.

Davis M, Rainnie D, Cassell M (1994) Neurotransmission in the rat amygdala related to fear and anxiety. Trends Neurosci 17:208-214.

Delaney AJ, Sah P (1999) GABA receptors inhibited by benzodiazepines mediate fast inhibitory transmission in the central amygdala. J Neurosci 19:9698-9704

Dettmer TS, Barnes A, Iqbal U, Bailey CD, Reynolds JN, Brien JF, Valenzuela CF (2003) Chronic prenatal ethanol exposure alters ionotropic glutamate receptor subunit protein levels in the adult guinea pig cerebral cortex. Alcohol Clin Exp Res 27:677-681.

Eckardt MJ, File SE, Gessa GL, Grant KA, Guerri C, Hoffman PL, Kalant H, Koob GF, Li TK, Tabakoff B (1998) Effects of moderate alcohol consumption on the central nervous system. Alcohol Clin Exp Res 22:998-1040.

Frantz KJ, Hansson KJ, Stouffer DG, Parsons LH (2002) 5-HT 6 receptor antagonism potentiates the behavioral and neurochemical effects of amphetamine but not cocaine. Neuropharmacology 42:170-180.

Gonzales RA, Brown LM (1995) Brain regional differences in glycine rever- 
sal of ethanol-induced inhibition of $N$-methyl-D-aspartate-stimulated neurotransmitter release. Life Sci 56:571-577.

Gonzales R, Tang A, Robinson D (2002) Quantitative microdialysis for in vivo studies of pharmacodynamics. In: Methods in alcohol-related neuroscience research (Liu Y, Lovinger D, eds), pp 287-317. New York: CRC.

Hoffman PL, Rabe CS, Moses F, Tabakoff B (1989) N-Methyl-D-aspartate receptors and ethanol: inhibition of calcium flux and cyclic GMP production. J Neurochem 52:1937-1940.

Hyytia P, Koob GF (1995) GABAA receptor antagonism in the extended amygdala decreases ethanol self-administration in rats. Eur J Pharmacol 283:151-159.

Kalivas PW, Alesdatter JE (1993) Involvement of N-methyl-D-aspartate receptor stimulation in the ventral tegmental area and amygdala in behavioral sensitization to cocaine. J Pharmacol Exp Ther 267:486-495.

Kalluri HS, Mehta AK, Ticku MK (1998) Up-regulation of NMDA receptor subunits in rat brain following chronic ethanol treatment. Brain Res Mol Brain Res 58:221-224.

Koltchine V, Anantharam V, Wilson A, Bayley H, Treistman SN (1993) Homomeric assemblies of NMDAR1 splice variants are sensitive to ethanol. Neurosci Lett 152:13-16.

Koob GF, Le Moal M (2001) Drug addiction, dysregulation of reward, and allostasis. Neuropsychopharmacology 24:97-129.

Koob GF, Roberts AJ, Schulteis G, Parsons LH, Heyser CJ, Hyytia P, MerloPich E, Weiss F (1998) Neurocircuitry targets in ethanol reward and dependence. Alcohol Clin Exp Res 22:3-9.

Krystal JH, Petrakis IL, Mason G, Trevisan L, D'Souza DC (2003) $\mathrm{N}$-Methyl-D-aspartate glutamate receptors and alcoholism: reward, dependence, treatment, and vulnerability. Pharmacol Ther 99:79-94.

Kumari M, Ticku MK (2000) Regulation of NMDA receptors by ethanol. Prog Drug Res 54:152-189.

Kumari M, Anji A, Woods Jr H, Ticku MK (2003) The molecular effects of alcohol: clues to the enigmatic action of alcohol. Ann NY Acad Sci 993: 82-94, 123-124.

Kuner T, Schoepfer R, Korpi ER (1993) Ethanol inhibits glutamate-induced currents in heteromeric NMDA receptor subtypes. NeuroReport 5:297-300.

Little HJ (1999) The contribution of electrophysiology to knowledge of the acute and chronic effects of ethanol. Pharmacol Ther 84:333-353.

Lopez de Armentia M, Sah P (2003) Development and subunit composition of synaptic NMDA receptors in the amygdala: NR2B synapses in the adult central amygdala. J Neurosci 23:6876-6883.

Lovinger DM (1995) Developmental decrease in ethanol inhibition of $\mathrm{N}$-methyl-D-aspartate receptors in rat neocortical neurons: relation to the actions of ifenprodil. J Pharmacol Exp Ther 274:164-172.

Lovinger DM (1997) Alcohols and neurotransmitter gated ion channels: past, present and future. Naunyn Schmiedebergs Arch Pharmacol 356:267-282.

Lovinger DM (2002) NMDA receptors lose their inhibitions. Nat Neurosci 5:614-616

Lovinger DM, White G, Weight FF (1989) Ethanol inhibits NMDAactivated ion current in hippocampal neurons. Science 243:1721-1724.

Macey DJ, Schulteis G, Heinrichs SC, Koob GF (1996) Time-dependent quantifiable withdrawal from ethanol in the rat: effect of method of dependence induction. Alcohol 13:163-170.

Maldve RE, Zhang TA, Ferrani-Kile K, Schreiber SS, Lippmann MJ, Snyder GL, Fienberg AA, Leslie SW, Gonzales RA, Morrisett RA (2002) DARPP-32 and regulation of the ethanol sensitivity of NMDA receptors in the nucleus accumbens. Nat Neurosci 5:641-648.

Martin D, Tayyeb MI, Swartzwelder HS (1995) Ethanol inhibition of AMPA and kainate receptor-mediated depolarizations of hippocampal area CA1. Alcohol Clin Exp Res 19:1312-1316.

Masood K, Wu C, Brauneis U, Weight FF (1994) Differential ethanol sensitivity of recombinant $N$-methyl-D-aspartate receptor subunits. Mol Pharmacol 45:324-329.

Mennerick S, Zorumski CF (1995) Paired-pulse modulation of fast excitatory synaptic currents in microcultures of rat hippocampal neurons. J Physiol (Lond) 488:85-101.

Meyer PJ, Phillips TJ (2003) Bivalent effects of MK-801 on ethanol-induced sensitization do not parallel its effects on ethanol-induced tolerance. Behav Neurosci 117:641-649.

Mirshahi T, Woodward JJ (1995) Ethanol sensitivity of heteromeric NMDA receptors: effects of subunit assembly, glycine and NMDAR1 $\mathrm{Mg}(2+)-$ insensitive mutants. Neuropharmacology 34:347-355.

Moghaddam B, Bolinao ML (1994) Biphasic effect of ethanol on extracellular accumulation of glutamate in the hippocampus and the nucleus accumbens. Neurosci Lett 178:99-102.

Nie Z, Madamba SG, Siggins GR (1994) Ethanol inhibits glutamatergic neurotransmission in nucleus accumbens neurons by multiple mechanisms. J Pharmacol Exp Ther 271:1566-1573.

Nose I, Higashi H, Inokuchi H, Nishi S (1991) Synaptic responses of guinea pig and rat central amygdala neurons in vitro. J Neurophysiol 65:1227-1241.

Paxinos G, Watson C (1986) The rat brain in sterotaxic coordinates. San Diego: Academic.

Pich EM, Lorang M, Yeganeh M, Rodriguez de Fonseca F, Raber J, Koob GF, Weiss F (1995) Increase of extracellular corticotropin-releasing factorlike immunoreactivity levels in the amygdala of awake rats during restraint stress and ethanol withdrawal as measured by microdialysis. J Neurosci 15:5439-5447.

Post RM, Weiss SR, Pert A (1987) The role of context and conditioning in behavioral sensitization to cocaine. Psychopharmacol Bull 23:425-429.

Quadros IM, Hipolide DC, Frussa-Filho R, De Lucca EM, Nobrega JN, SouzaFormigoni ML (2002) Resistance to ethanol sensitization is associated with increased NMDA receptor binding in specific brain areas. Eur J Pharmacol 442:55-61.

Quertemont E, de Neuville J, De Witte P (1998) Changes in the amygdala amino acid microdialysate after conditioning with a cue associated with ethanol. Psychopharmacology 139:71-78.

Rassnick S, Heinrichs SC, Britton KT, Koob GF (1993) Microinjection of a corticotropin-releasing factor antagonist into the central nucleus of the amygdala reverses angiogenic-like effects of ethanol withdrawal. Brain Res 605:25-32.

Reynolds JD, Brien JF (1994) Effects of acute ethanol exposure on glutamate release in the hippocampus of the fetal and adult guinea pig. Alcohol 11:259-267.

Roberto M, Madamba SG, Moore SD, Tallent MK, Siggins GR (2003) Ethanol increases GABAergic transmission at both pre- and postsynaptic sites in rat central amygdala neurons. Proc Natl Acad Sci USA 100:2053-2058.

Roberts AJ, Cole M, Koob GF (1996) Intra-amygdala muscimol decreases operant ethanol self-administration in dependent rats. Alcohol Clin Exp Res 20:1289-1298.

Robinson DL, Lara JA, Brunner LJ, Gonzales RA (2000) Quantification of ethanol concentrations in the extracellular fluid of the rat brain: in vivo calibration of microdialysis probes. J Neurochem 75:1685-1693.

Robledo P, Robbins TW, Everitt BJ (1996) Effects of excitotoxic lesions of the central amygdaloid nucleus on the potentiation of reward-related stimuli by intra-accumbens amphetamine. Behav Neurosci 110:981-990.

Rogers J, Wiener SG, Bloom FE (1979) Long-term ethanol administration methods for rats: advantages of inhalation over intubation or liquid diets. Behav Neural Biol 27:466-486.

Rossetti ZL, Carboni S (1995) Ethanol withdrawal is associated with increased extracellular glutamate in the rat striatum. Eur J Pharmacol 283: 177-183.

Sah P, Lopez De Armentia M (2003) Excitatory synaptic transmission in the lateral and central amygdala. Ann NY Acad Sci 985:67-77.

Simson PE, Criswell HE, Breese GR (1993) Inhibition of NMDA-evoked electrophysiological activity by ethanol in selected brain regions: evidence for ethanol-sensitive and ethanol-insensitive NMDA-evoked responses. Brain Res 607:9-16.

Tabakoff B, Hoffman PL (1996) Alcohol addiction: an enigma among us. Neuron 16:909-912.

Timmerman W, Westerink BH (1997) Brain microdialysis of GABA and glutamate: what does it signify? Synapse 27:242-261.

Trevisan L, Fitzgerald LW, Brose N, Gasic GP, Heinemann SF, Duman RS, Nestler EJ (1994) Chronic ingestion of ethanol up-regulates NMDAR1 receptor subunit immunoreactivity in rat hippocampus. J Neurochem 62:1635-1638.

Tsai G (1998) Glutamatergic neurotransmission in alcoholism. J Biomed Sci 5:309-320.

Tsai G, Coyle JT (1998) The role of glutamatergic neurotransmission in the pathophysiology of alcoholism. Annu Rev Med 49:173-184. 
Vanderschuren LJ, Kalivas PW (2000) Alterations in dopaminergic and glutamatergic transmission in the induction and expression of behavioral sensitization: a critical review of preclinical studies. Psychopharmacology 151:99-120.

Wan FJ, Berton F, Madamba SG, Francesconi W, Siggins GR (1996) Low ethanol concentrations enhance GABAergic inhibitory postsynaptic potentials in hippocampal pyramidal neurons only after block of GABAB receptors. Proc Natl Acad Sci USA 93:5049-5054.

Watanabe T, Nakagawa T, Yamamoto R, Maeda A, Minami M, Satoh M (2002) Involvement of glutamate receptors within the central nucleus of the amygdala in naloxone-precipitated morphine withdrawal-induced conditioned place aversion in rats. Jpn J Pharmacol 88:399-406.

Williams K (2001) Ifenprodil, a novel NMDA receptor antagonist: site and mechanism of action. Curr Drug Targets 2:285-298.

Wolf ME, Dahlin SL, Hu XT, Xue CJ, White K (1995) Effects of lesions of prefrontal cortex, amygdala, or fornix on behavioral sensitization to amphetamine: comparison with $N$-methyl-D-aspartate antagonists. Neuroscience 69:417-439.

Woodward JJ (1999) Ionotropic glutamate receptors as sites of action for ethanol in the brain. Neurochem Int 35:107-113.

Woodward JJ (2000) Ethanol and NMDA receptor signaling. Crit Rev Neurobiol 14:69-89.

Xie ZC, Buckner E, Commissaris RL (1995) Anticonflict effect of MK-801 in rats: time course and chronic treatment studies. Pharmacol Biochem Behav 51:635-640.

Yaka R, Phamluong K, Ron D (2003) Scaffolding of Fyn kinase to the NMDA receptor determines brain region sensitivity to ethanol. J Neurosci 23:3623-3632.

Yang X, Criswell HE, Simson P, Moy S, Breese GR (1996) Evidence for a selective effect of ethanol on $\mathrm{N}$-methyl-D-aspartate responses: ethanol affects a subtype of the ifenprodil-sensitive $N$-methyl-D-aspartate receptors. J Pharmacol Exp Ther 278:114-124. 